\title{
The importance of antennae for pea aphid wing induction in the presence of natural enemies
}

\author{
G. Kunert* and W.W. Weisser \\ Institute of Ecology, Friedrich-Schiller University Jena, \\ Dornburger Str. 159, 07743 Jena, Germany
}

\begin{abstract}
The pea aphid Acyrthosiphon pisum Harris has been shown to produce an increasing proportion of winged morphs among its offspring when exposed to natural enemies, in particular hoverfly larvae, lacewing larvae, adult and larval ladybirds and aphidiid parasitoids. While these results suggest that wing induction in the presence of predators and parasitoids is a general response of the pea aphid, the cues and mechanisms underlying this response are still unclear. Tactile stimuli and the perception of chemical signals as well as visual signals are candidates for suitable cues in the presence of natural enemies. In this paper the hypothesis that the aphids' antennae are crucial for the wing induction in the presence of natural enemies is tested. Antennae of pea aphids were ablated and morph production was scored when aphids were reared either in the presence or the absence of predatory lacewing larvae over a six-day period. Ablation of antennae resulted in a drastic drop in the proportion of winged morphs among the offspring, both in the presence and the absence of a predator whereas predator presence increased wing induction in aphids with intact antennae, as reported in previous experiments. The results show that antennae are necessary for wing induction in the presence of natural enemies. Critical re-examination of early work on the importance of aphid antennae and tactile stimuli for wing induction suggests that a combination of tactile and chemical cues is likely to be involved not only in predator-induced wing formation but also for wing induction in response to factors such as crowding in the aphid colony.
\end{abstract}

\section{Introduction}

Aphids (Hemiptera: Aphididae) are the most important pest insects in the temperate regions, damaging plants by phloem sucking and the transmission of plant viruses (Minks \& Harrewijn, 1987). One characteristic feature of aphid life-cycles is cyclical parthenogenesis. Sexual reproduction takes place only in autumn, when males and females mate and produce a diapausing egg. From spring to autumn, aphids reproduce asexually such that all aphids descending from a female that hatches in spring are genetically identical (Dixon, 1998). During the asexual phase, many aphid species can produce two types of morphs: a winged morph that is

*Fax: ++49 (0) 3641949402

E-mail: Grit.Kunert@uni-jena.de mainly responsible for dispersal and the colonization of new plants, and an unwinged morph that mostly stays on the plant on which it was born. Importantly, in many species such as Acyrthosiphon pisum it is the aphid mother that either partly or completely determines the phenotype of the offspring (Kawada, 1987).

For almost as long as it has been known that virginoparous aphids can produce winged or unwinged offspring, researchers have tried to find the cues and mechanism underlying this wing polyphenism (Kawada, 1987; Dixon, 1998; Müller et al., 2001). Early investigations revealed the importance of photoperiod (e.g. Bonnemaison, 1951; Lees, 1966) and host plant quality (Mittler \& Sutherland, 1969; Sutherland, 1969b; Dixon \& Glen, 1971) for the induction of winged morphs. Several experiments showed that wing induction was suppressed in the presence of tending ants (Hille Ris Lambers, 1966). In addition, a number of 
a) induced by crowding b) induced by ladybird larvae c) induced by natural enemies



Fig. 1. Comparison of proposed mechanisms of aphid wing induction by crowding and natural enemies. a) Induction solely by tactile stimuli in Acyrthosiphon pisum (Sutherland, 1969), Megoura viciae (Lees, 1967), Aphis craccivora (Johnson, 1965); b) Induction by the presence of ladybird larvae in A. pisum (Dixon \& Agarwala, 1999); c) Induction by the presence of natural enemies in A. pisum (Weisser et al., 2004; Sloggett \& Weisser, 2004).

experiments showed that winged morphs were born when aphids were reared under crowded conditions (Johnson, 1965; Lees, 1967; Sutherland, 1969a; Shaw, 1970; Mittler \& Kunkel, 1971; Sutherland \& Mittler, 1971). While the crowding experiments identified the environmental conditions under which alatae or apterae were born, they did not conclusively identify how wing induction occurs under crowding conditions. Nutritional quality and a visual stimulus were thought to be possible cues for wing induction. Visual cues were, however, ruled out in further experiments (Johnson, 1965; Lees, 1967), whereas no clear pattern for the relationship between nutrition and wing induction emerged (Lees, 1967; Sutherland, 1969b; Müller et al., 2001; Sloggett \& Weisser, 2004) possibly because different aphid species use different cues to perceive plant quality. Other possibilities were tactile and olfactory stimuli. For Megoura viciae Buckton (Lees, 1967) and Aphis craccivora Koch (Johnson, 1965), olfactory stimuli could be excluded because crowded aphids, without antennae, which bear the only known sensoria for volatile chemicals in aphids, still produced an increased proportion of winged morphs among their offspring. Because tactile stimuli are perceived by tactile bristles and hairs on the legs, body and also the antennae of aphids (Minks \& Harrewijn, 1987), the amputation of the antennae does not rule out the involvement of tactile stimuli. Pea aphids Acyrthosiphon pisum Harris with ablated antennae, did not, however, react to crowding stimuli (Sutherland, 1969a). Sutherland's reasoning was that possibly the most important mechanoreceptors in A. pisum are located on the antennae. Because the antennae also bear chemoreceptors it was still unclear what stimuli triggered the crowding response in A. pisum. To solve this puzzle, Sutherland (1969a) performed some investigations, caging single $A$. pisum in small muslin bags for $24 \mathrm{~h}$ in a way that either the aphids could walk freely but the aphid's antennae had continuous contact with the wall of the muslin bag, or the bag was so small that the aphids were unable to move at all. $100 \%$ of the walking aphids became alatae producers whereas only $35 \%$ of the aphids in the smaller bags produced alate offspring. Since the aphids in the small bags had no contact with aphids other than their own small group of offspring, Sutherland (1969a) concluded that tactile stimuli together with aphid movement are the main cues (fig. 1a). At the beginning of the 1970s, it therefore seemed that the major environmental factor involved in aphid wing polyphenism-the tactile cue-had been unravelled along with most of the underlying mechanisms. But it must be pointed out that even though the investigated aphid species were all responsive to tactile stimuli in some way they perceived this stimulus with different parts of their body, as evidenced by the different responses of the different species to antennal ablation. The role of contact chemical cues remains entirely unstudied to date, i.e. chemical recognition that may occur when aphids touch one another, which occurs in other insect species (e.g. Chapman, 1998; Wagner et al., 2000; Cervo et al., 2002; Ruther et al., 2002; Ginzel \& Hanks, 2003; Kaib et al., 2004). Nevertheless for the next 30 years, experimentation on this subject largely stopped (but see e.g. Watt \& Dixon, 1981; review in Kawada, 1987).

Recently, it was found that pea aphids increase winged morph production in the presence of natural enemies (Weisser et al., 1999; Dixon \& Agarwala, 1999; Sloggett \& Weisser, 2002; Kunert \& Weisser, 2003) and the question about the mechanisms and cues was raised again (Sloggett \& Weisser, 2004). Although it is known that tactile cues are most important for wing induction caused by crowding, it is not at all clear if the same cues and mechanisms are involved in wing induction caused by natural enemies. When predators are searching in an aphid colony, a number of potential cues are available that are not present when aphids are crowded. For example, potential cues which emanate from the predator individual itself (predator smell, visual cue) are obviously not present in crowded colonies without predators and could be involved in wing induction. Similarly, indirect cues signalling the presence of a predator that emanate from attacked aphids are also not present in crowded colonies. These cues must be ruled out and the cues involved in wing induction under crowding and starvation must be tested again before it can be concluded that wing induction in the presence of predators is similar to wing induction under crowding and changed host plant quality. It is also necessary 
to allow for the possibility that a combination of cues leads to wing induction.

Thus Dixon \& Agarwala (1999) performed experiments with single $A$. pisum put on plants or into tubes previously exposed to ladybird larvae or the aphid-predator complex, thereby excluding tactile stimuli to predators or aphids but not to the plant or tube wall. Dixon \& Agarwala (1999) found that aphids put on contaminated plants or into contaminated tubes produced a higher proportion of winged morphs than control aphids and concluded that ladybird larval tracks were responsible for wing induction (fig. 1b) but theoretically, other substances emitted by the predator or the aphidpredator complex could also have been involved (Weisser et al., 2004). Also, the importance of a visual cue was not ruled out because the aphids had no possibility of seeing the predator, and the involvement of visual cues was therefore not tested. That ladybird larval tracks might not be the only important cue for wing induction is supported by experiments without ladybird larvae but with ladybird adults or with other predators or parasitoids searching in aphid colonies which also led to an increasing proportion in winged offspring (Weisser et al., 1999; Sloggett \& Weisser, 2002; Kunert \& Weisser, 2003). Thus general visual cues, such as a perception of defence behaviour in other aphids, or general chemical signals, such as aphid alarm pheromone (Bowers et al., 1972; Sloggett \& Weisser, 2004), or even a general enemy odour or enemy tracks, might lead to wing induction (fig. 1c). In addition, any of these cues might lead to dropping or walking of aphids and therefore result in an increased contact rate and tactile stimulation among aphids which then results in wing induction. Thus a cascade of signals is possible, starting with direct disturbance caused by the enemy directly or indirectly promoting an increasing amount of contact among individuals which then results in wing induction (fig. 1c).

Visual cues can only be perceived by the eyes and ocelli. Tactile stimuli can be perceived in aphids by tactile hairs and bristles on the head, body, and especially the legs. Volatiles such as aphid alarm pheromone (E)- $\beta$-farnesene can only be perceived by the rhinaria on the aphids' antennae (Bowers et al., 1972; Nault et al., 1973). Finally, perception of contact chemicals is poorly studied in aphids but in insects contact chemical sensilla can be found on different parts of the body such as the mouthparts, tarsi, ovipositor, and antennae (Chapman, 1998). To find out which cue might trigger wing induction it is convenient to confine the possibilities by investigating the body part which is important for the perception of the cue. Since excision of the antennae (removal of the primary and secondary rhinaria) has been shown to completely eliminate the response to cornicle droplets in A. pisum, Acyrthosiphon solani Kaltenbach, and Myzus persicae Sulzer (Nault et al., 1973), it may be concluded that antennal ablation is a suitable method to exclude the role of alarm pheromone and other volatile chemicals for aphid wing induction in the presence of natural enemies. However, it does not rule out the involvement of tactile stimuli which can also be perceived by tactile hairs and bristles on the head, body, and especially legs of an aphid. Antennal ablation also allows conclusions to be drawn about the importance of visual stimuli, and of tactile and chemical sensilla on other parts of the body.

In this paper the importance of the antennae for wing induction in aphids in the presence of natural enemies is investigated by exposing aphids with and without antennae to predatory lacewing larvae. If aphids with amputated antennae still respond to the presence of a predator, volatile chemical cues can be excluded. The focus must then switch to the visual cues or to sensilla for perception of tactile stimuli, or contact chemical stimuli on other parts of the body.

\section{Materials and methods \\ Experimental animals and plants}

For the experiment, the red clone BP of the pea aphid A. pisum was used. This clone originated in Bayreuth (Germany) and was used in a number of related studies (Weisser et al., 1999; Sloggett \& Weisser, 2002; Kunert \& Weisser, 2003). The aphids were reared and the experiment was conducted on three-week-old broad bean plants Vicia faba L.; variety 'The Sutton' (Nickerson-Zwaan, UK), approximately $10-15 \mathrm{~cm}$ high with $4-5$ leaf pairs. The plants were cultivated separately in $10-\mathrm{cm}$ diameter flowerpots. In order to prevent the aphids and predators from escaping, each plant was covered with an air-permeable cellophane bag $(18.5 \mathrm{~cm} \times 39 \mathrm{~cm})$.

Lacewing larvae Chrysoperla carnea Stephens sensu lato (Neuroptera: Chrysopidae) were obtained from a commercial supplier (Katz Biotech Services, Welzheim, Germany). The larvae were reared on bean plants infested with pea aphids until they reached the second larval stage. They were then used for the experiment. Plants, aphids and lacewing larvae were raised under the same conditions (16 h light, $8 \mathrm{~h}$ dark, $20^{\circ} \mathrm{C}, 75 \%$ relative humidity).

\section{Experimental design}

The experiment took place under the same conditions as the rearing of the insect cultures.

For the experiment, 24 lines of aphids were established. Each line was used for one replication. To initiate a line, one single foundress was placed on a bean plant and left to reproduce for two days. The offspring (grandparents) remained on the plant and were grown until they reached the young adult stage, when they were transferred separately to new bean plants to avoid crowding. After two days, when the aphids had given birth several times, the adults were removed and the progeny (parents) were reared until the fourth larval stage and then used for the experiment. For the experiment the parents of each line were divided into four groups of 15 aphids each for the following four treatments:

1. 15 unmanipulated aphids with one lacewing larva (+ antennae + predator)

2. 15 aphids with amputated antennae with one lacewing larva $(-\mathrm{a}+\mathrm{p})$

3. 15 unmanipulated aphids without a lacewing larva $(+\mathrm{a}$ $-\mathrm{p})$

4. 15 aphids with amputated antennae without a lacewing larva $(-\mathrm{a}-\mathrm{p})$

For antennal amputation, the aphids were anaesthetized with carbon dioxide for, on average, $3 \mathrm{~s}$ until they did not move anymore. Then the antennae were cut between the second and third segment to remove all rhinaria important for detection of volatiles. The removal of the antennae was performed under a binocular microscope using a fine scalpel. 
In order to prevent a biased result because of possible effects of anaesthetization, aphids of all treatments were anaesthetized and handled in the same way as in the treatments with amputated antennae, except for the actual ablation of the antennae. Afterwards the aphids were placed in groups of five on the top two leaf pairs of the plant. In predator treatments, one lacewing larva was placed on the lowest leaf pair of the plant to give aphids time to recover before first contact with a predator larva. After three days, which represents the first period of the experiment, the parents had given birth to a number of offspring. The parents were transferred to new bean plants to avoid overcrowding and early death of plants and aphids. The offspring were reared for a number of further days on the same plants, in the predator treatment the lacewing larvae were taken off the plant. New second instar lacewing larvae were introduced to the predator treatments on the new plants. After three further days, the second period of the experiment, the parents and the predators were removed from the second set of plants and the offspring produced during this period were also reared for a number of days on the plants. Whereas the morph of the offspring born during the first period may have been determined before the treatment started (Sutherland, 1969a), the morphs of the offspring born during the second period were only influenced by the treatment.

At the end of each 3-day period the number of surviving adult aphids was recorded. All offspring produced by the adult aphids during the experiment were reared until they reached the fourth instar or early adult stage, then taken off the plant and frozen for later counting and determination of the phenotype. In fourth instar larvae the wing buds indicating a winged morph can easily be identified.

\section{Statistical analysis}

Results are presented as means \pm standard error in all cases. For the comparison of proportional data (survival of the adults, percentage of winged offspring) a generalized linear model was used, simplified by removing nonsignificant terms (Crawley, 2002). The analyses were done in the statistical program R, version 1.6.2 (Venables et al., 2002). Box plots were used to show differences between treatments. These display the 25th and 75th percentiles as lines on a bar centred about the median, and the 10th and 90th percentiles as error bars. Outliers are shown as dots.

For the comparison of numbers of aphids, two-way ANOVAs were used after testing for normality and equal variances. For these analyses the software package SigmaStat for Windows, version 2.03 was utilized.

\section{Results \\ Survival of the adult aphids}

In both 3-day periods the maximum survival of parental aphids for all treatments was $100 \%$ (fig. 2), and the minimum survival was $0 \%$ in the treatment of unmanipulated aphids with predator $(+\mathrm{a}+\mathrm{p})$ in period 2. Survival was lower if the adult aphids were exposed to a lacewing larva and slightly lower if the aphids had no antennae (fig. 2, period 1: d.f. $=93$, predator: $\mathrm{t}=-3.94, P<0.001$, antennae: $\mathrm{t}=2.36$, $P<0.05$; period 2: d.f. $=93$, predator: $\mathrm{t}=-5.61, P<0.001$, antennae: $\mathrm{t}=2.31, P<0.05)$. The interaction between predator and aphids with antennae was not significant for either
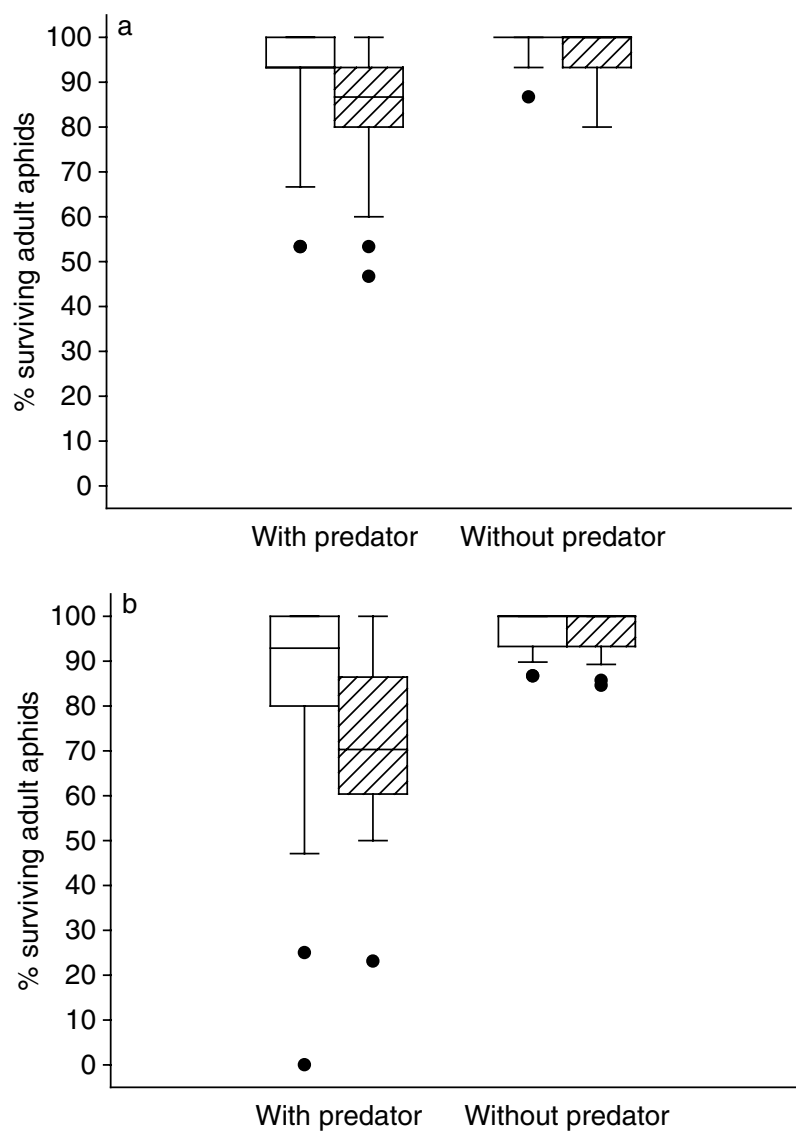

Fig. 2. Survival of adult Acyrthosiphon pisum of the parental generation. a) Period 1 (days 1-3), b) period 2 (days 4-6, aphids were transferred to new plants at day 4). Open bars, aphids with antennae; hatched bars, aphids without antennae. Lines centred about the median are the 25th and 75th percentiles, error bars are 10 th and 90th percentiles. Dots are outliers.

period (period 1: d.f. $=92, \mathrm{t}=-0.69, \mathrm{NS}$; period 2: d.f. $=92$, $\mathrm{t}=1.18$, NS) and was therefore removed from the model.

\section{Offspring number}

The data from one aphid line of the first period was discarded, because the predator was overlooked when the adult aphids were removed and it continued to prey on the offspring while these were reared to adulthood.

The total number of offspring counted at the end of the experiment was significantly lower in the treatments with predator than in the no-predator treatments, both in the first and in the second period (fig. 3, period 1: d.f. $=91, \mathrm{~F}=10.60$, $P<0.01$; period 2: d.f. $=95, \mathrm{~F}=28.84, P<0.001)$. There was no significant influence of antennal amputation on offspring number (period 1: d.f. $=91, \mathrm{~F}=1.48, \mathrm{NS}$; period 2: d.f. $=95$, $\mathrm{F}=3.44, \mathrm{NS}$ ) and the interaction between predator and antennal treatments was also not significant (period 1: d.f. $=91, \mathrm{~F}=2.84$, NS; period 2: d.f. $=95, \mathrm{~F}=0.15$, NS).

\section{Importance of the antennae for wing induction}

In the first period, the ablation of antennae significantly reduced the percentage of winged morphs among the 

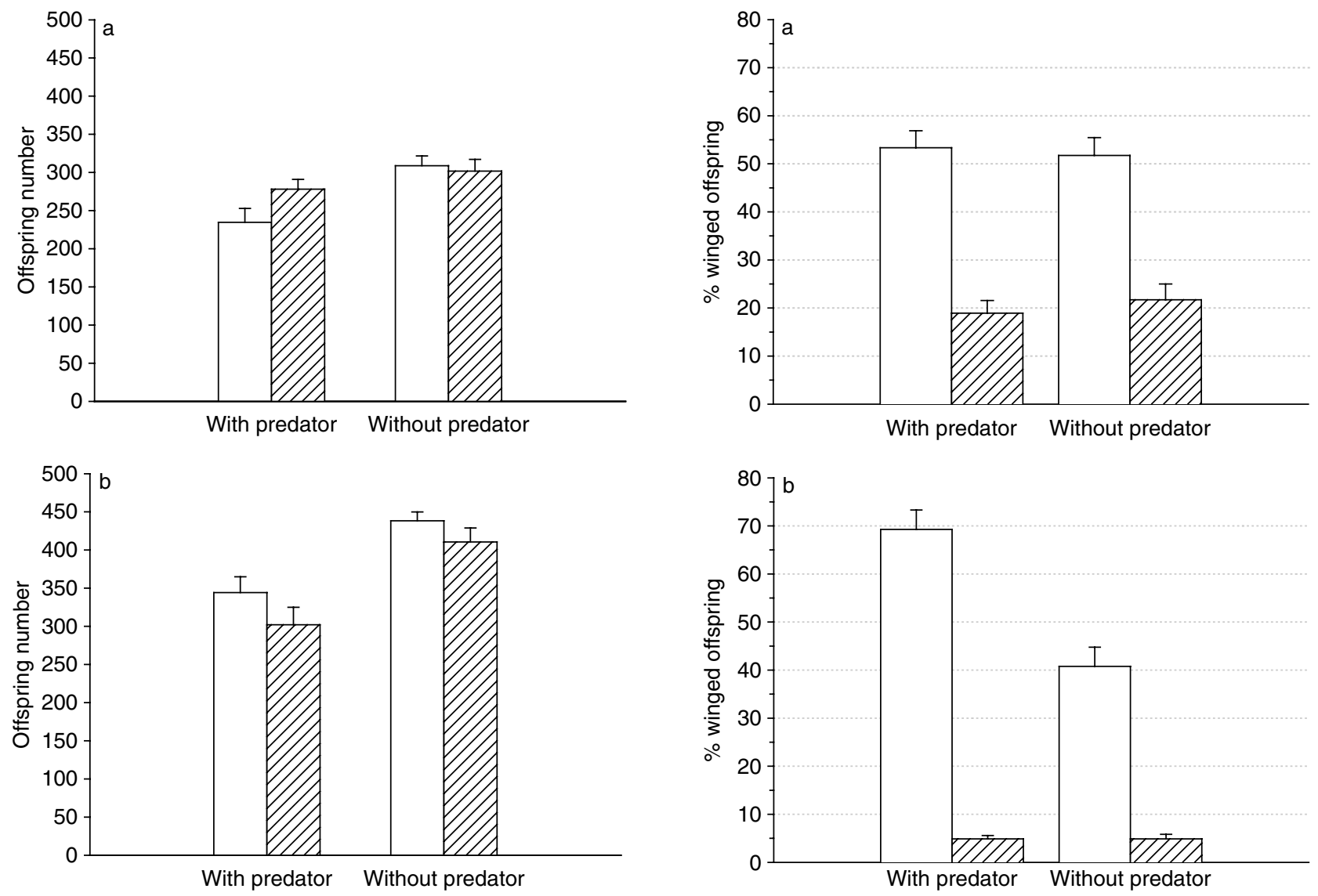

Fig. 3. Number of offspring of Acyrthosiphon pisum produced during the experiment. a) Period 1 (days 1-3), b) period 2 (days 4-6, aphids were transferred to new plants at day 4). Open bars, parent generation with antennae; hatched bars, parent generation without antennae.

offspring (d.f. $=90, t=9.53, P<0.001$; fig. $4 a$ ) while the presence of predators had no effect on winged morph induction (d.f. $=89, \mathrm{t}=-0.08$, NS). The interaction between predator and antennal treatments was also not significant (d.f. $=88, \mathrm{t}=0.90, \mathrm{NS}$ ). Both coefficients (predator and interaction) were therefore removed from the model.

In the second period, removal of the antennae also significantly reduced the percentage of winged offspring (d.f. $=92, \mathrm{t}=9.10, P<0.001$; fig. $4 \mathrm{~b}$ ). While the main effect of predator presence was not significant (d.f. $=92, t=0.21$, NS), the interaction between the predator and antennal treatments was significant (d.f. $=92, \mathrm{t}=2.55, P<0.05$ ). In the treatments with amputated antennae the percentage of winged morphs among the offspring was very low, independent of the presence of the predator (fig. $4 \mathrm{~b}$ ). In the treatments with unmanipulated aphids the presence of the predator greatly increased the occurrence of winged morphs (fig. 4b).

\section{Discussion}

The results clearly indicate that the antennae of A. pisum are of particular importance for wing induction caused by

Fig. 4. Mean proportion of winged morphs of Acyrthosiphon pisum among the offspring produced during the experiment. a) Period 1 (days 1-3), b) period 2 (days 4-6, aphids were transferred to new plants at day 4). Open bars, parent generation with antennae; hatched bars, parent generation without antennae.

natural enemies. In the presence of predators, very few winged offspring were produced by aphids without antennae. Thus, the involvement of a volatile chemical cue in aphid wing induction in the presence of a predator cannot be ruled out. In contrast, this experiment clearly rules out the involvement of visual cues as the individuals with ablated antennae were still able to see their environment. With respect to tactile or contact chemical cues, the experiment leads to the conclusion that for pea aphids, tactile hairs and bristles and contact chemical sensilla on the rest of the body are not of prime importance for wing induction.

One potential problem of experiments where body parts are excised or manipulated is that the manipulation causes malfunction in the organisms in a way that would not normally occur in intact individuals. In this case here, there is the possibility that antennal amputation caused aphids to be unable to produce winged offspring independent of any cues perceived. This is considered to be very unlikely. The ablation of the antennae did not have a significant influence on the number of offspring born, and the survival of aphids without antennae was only slightly lower than the survival of unmanipulated aphids in the predator treatment, and 
identical in the no-predator treatment. In addition, A. pisum without antennae did produce some winged offspring, showing that the production of winged individuals was not physiologically impossible. In M. viciae (Lees, 1967) and A. craccivora (Johnson, 1965) it has also been shown that aphids without antennae can produce winged offspring. Finally, the very low percentage of winged offspring with manipulated aphids in the predator treatment cannot be due to low activity of the predator, because the predator significantly influenced the number of surviving adult aphids as well as the number of offspring produced.

Further support that the experimental design was appropriate comes from the significant effect of predators on winged morph induction among the unmanipulated A. pisum in the second period which mirrors the effects seen in previous experiments (Kunert \& Weisser, 2003). That there was no predator effect in the first period might be due to aphid handling. The anaesthetization and especially the grouping of always five aphids on one leaf may have lead to something similar to crowding when the aphids recovered from anaesthetization, resulting in an increased proportion of winged morphs in all treatments. This effect possibly superseded the effect of the predator, which is normally quite small in the first period (Kunert \& Weisser, 2003). In the treatments with ablated antennae, the proportion of winged morphs was higher in the first than in the second period. A likely explanation is that some of these offspring were determined before the experiment started. The percentage of winged offspring under control conditions, without any further disturbance, is about 30-40\% (Kunert \& Weisser, 2003).

The possibility that tactile cues are perceived differently in different aphid species has not been considered much in the literature (e.g. Müller et al., 2001), i.e. the implicit assumption has been that tactile cues can be perceived in all aphids by tactile hairs that are present at several places on the aphid body, not only on the antennae. If this is true, however, the only possible reason for the low percentage of winged offspring of manipulated aphids in the present experiment would be that the perception of the cue in the pea aphid is confined to sensory organs that only occur on the antennae, which implies that the cue must be chemical. A similarity in the perception of tactile cues across aphid species would also challenge the standard interpretation of the results of the pioneering experiments on wing induction in the pea aphid by Sutherland (1969a). Because Sutherland (1969a) showed that A. pisum with amputated antennae needed the antennae to produce a higher proportion of winged offspring when crowded, the implication would be that, at least in pea aphids, the reaction to crowding would also involve a chemical cue. Sutherland's (1969a) experimental procedure, although quite ingenious, does, in fact, leave room for alternative explanations. Sutherland (1969a) caged single aphids in small muslin bags (1.5 inches long and $<1$ inches in diameter for $24 \mathrm{~h}$ ). The resulting intense stimulation of bristles and hairs within these muslin bags is likely to be very different from the natural situation, where under crowded conditions aphids would repeatedly but probably not constantly touch one another with their antennae or other body parts. Thus it remains unclear whether these stimuli were unreasonable, i.e. not representing adequately what happens in a crowded colony. As discussed in the introduction, it cannot be ruled out that chemical recognition, analogous to kin or mate recognition shown for other insect species, rather than the pure contact of tactile hairs to another object, is involved in aphid wing induction. This possibility was mentioned by Sutherland (1969a), but was rejected because he considered it to be unlikely that different aphid species use different cues. If aphids react to compounds on the surface of other aphids, the intense contact to the offspring born in the small muslin bag (and to its own cuticula in the confined space of the bag) may have been enough for wing induction, independent of the perception of tactile cues. In addition, when an aphid walks in a small muslin bag, it is possible that the surface chemicals cover the hairs on the inside of the muslin bag and are subsequently detected by the aphid touching this surface. Note that this would also explain why aphids that were walking in the bag more often became alatae producers than aphids which were not able to move in the bag. Thus, Sutherland's results are entirely consistent with both the possibility of a volatile chemical cue, or a contact chemical cue, not only with tactile cues.

The possibility that chemicals are an important cue for wing induction in pea aphids, for crowding as well as for predator-induced wing production, is intriguing. However, the experiments with M. viciae (Lees, 1967) and A. craccivora (Johnson, 1965) have conclusively shown that in these species tactile stimulation alone can result in increased winged morph production. Thus, there is the possibility that A. pisum needs the tactile hairs on the antennae, apart from those on the head, body and legs, to perceive a sufficiently large tactile stimulus for wing induction. Because it is known that M. viciae (Lees, 1967) and A. craccivora (Johnson, 1965) do not need antennae for perception of tactile stimuli, this would imply that the perception of tactile stimuli may be different for different aphid species. That $A$. pisum perceives tactile stimuli with more involvement of the antennae than $M$. viciae is supported by the fact that $A$. pisum has more hairs on the antennae than $M$. viciae (Kunert, unpublished data). But on the other hand, the length of these hairs, which may also influence the perception of tactile stimuli, is greater in $M$. viciae than in A. pisum (Kunert, unpublished data). Thus, the possibility that crowded pea aphids react to tactile stimuli rather than to a chemical cue but perceive these stimuli with more involvement of the antennae than in $M$. viciae and A. craccivora seems more parsimonious than to assume a chemical cue for crowding. However, until further proof is brought forward, it is not possible to distinguish between these possibilities. These considerations show that after a 30-year period with little research activity on aphid wing polyphenism, more experiments are necessary to determine the way in which tactile stimuli are perceived in aphids.

To summarize, the above discussion demonstrates that in the regulation of aphid polyphenism both the perception of environmental stimuli and the reaction to different environmental stimuli may differ among species in ways that have not been investigated in detail so far. Whether tactile or chemical cues, or both, are responsible for wing induction in the presence of natural enemies requires more manipulative experiments with different aphid species. Similarly, if it can be conclusively shown that chemical cues are solely responsible for wing induction, the nature of this cue needs to be determined. Thus, the finding that $A$. pisum produce winged morphs in the presence of natural enemies has brought a new impetus to the old search for proximate mechanisms in aphid wing polyphenism. 


\section{Acknowledgements}

The authors are very grateful to John Sloggett for helpful discussions and for his comments to the manuscript. They would also like to thank Katz Biotech Services for donating C. carnea, and Ingrid Jakobi for technical assistance. This work was supported by grant WE 2816/2-1 of the Deutsche Forschungsgemeinschaft (DFG).

\section{References}

Bonnemaison, L. (1951) Contribution á l'etude des facteurs provoquant 1'apparition des formes ailées les Aphidinae. Annals des Epiphyties 2, 1-380.

Bowers, W.S., Webb, R.E., Nault, L.R. \& Dutky, S.R. (1972) Aphid alarm pheromone: isolation, identification, synthesis. Science 177, 1121-1122.

Cervo, R., Dani, F.R., Zanetti, P., Massolo, A. \& Turillazzi, S. (2002) Chemical nestmate recognition in a stenogastrine wasp, Liostenogaster flavolineata (Hymenoptera: Vespidae). Ethology, Ecology and Evolution 14, 351-363.

Chapman, R.F. (1998) The insects - structure and function. 4th edn. Cambridge, Cambridge University Press.

Crawley, M.J. (2002) Statistical computing. Chichester, West Sussex, John Wiley \& Sons Ltd.

Dixon, A.F.G. (1998) Aphid ecology. London, Chapman \& Hall.

Dixon, A.F.G. \& Agarwala, B.K. (1999) Ladybird-induced lifehistory changes in aphids. Proceedings of the Royal Society of London Series B 266, 1549-1553.

Dixon, A.F.G. \& Glen, D.M. (1971) Morph determination in the bird cherry-oat aphid Rhopalosiphum padi L. Annals of Applied Biology 68, 11-21.

Ginzel, M.D. \& Hanks, L.M. (2003) Contact pheromones as mate recognition cues of four species of longhorned beetles (Coleoptera: Cerambycidae). Journal of Insect Behavior 16, 181-187.

Hille Ris Lambers, D. (1966) Polymorphism in Aphididae. Annual Review of Entomology 11, 47-78.

Johnson, B. (1965) Wing polymorphism in aphids II. Interaction between aphids. Entomologia Experimentalis et Applicata 8, 49-64.

Kaib, M., Jmhasly, P., Wilfert, L., Durka, W., Franke, S., Francke, W., Leuthold, R.H. \& Brandl, R. (2004) Cuticular hydrocarbons and aggression in the termite Macrotermes subhyalinus. Journal of Chemical Ecology 30, 365-385.

Kawada, K. (1987) Polymorphism and morph determination. pp. 299-314 in Minks, A.K. \& Harrewijn, P. (Eds) Aphids, their biology, natural enemies and control. Vol. A. Amsterdam, Elsevier.

Kunert, G. \& Weisser, W.W. (2003) The interplay between density- and trait-mediated effects in predator-prey interactions: a case study in aphid wing polymorphism. Oecologia 135, 304-312.

Lees, A.D. (1966) The control of polymorphisms in aphids. Advances in Insect Physiology 3, 207-277.

Lees, A.D. (1967) The production of the apterous and alate forms in the aphid Megoura viciae Buckton, with special reference to the role of crowding. Journal of Insect Physiology 13, 289-318.

Minks, A.K. \& Harrewijn, P. (1987) Aphids, their biology, natural enemies and control. Vol. A. Amsterdam, Elsevier.
Mittler, T.E. \& Kunkel, H. (1971) Wing production by grouped and isolated apterae of the aphid Myzus persicae on artificial diet. Entomologia Experimentalis et Applicata 14, 83-92.

Mittler, T.E. \& Sutherland, O.R.W. (1969) Dietary influences on aphid polymorphism. Entomologia Experimentalis et Applicata 12, 703-713.

Müller, C.B., Williams, I.S. \& Hardie, J. (2001) The role of nutrition, crowding and interspecific interactions in the development of winged aphids. Ecological Entomology 26, 330-340.

Nault, L.R., Edwards, L.J. \& Styer, W.E. (1973) Aphid alarm pheromones: secretion and reception. Environmental Entomology 2, 101-105.

Ruther, J., Sieben, S. \& Schricker, B. (2002) Nestmate recognition in social wasps: manipulation of hydrocarbon profiles induces aggression in the European hornet. Naturwissenschaften 89, 111-114.

Shaw, M.J.P. (1970) Effects of population density on aliencolae of Aphis fabae Scop. II. The effects of crowding on the expression of migratory urge among alatae in the laboratory. Annals of Applied Biology 65, 197-203.

Sloggett, J.J. \& Weisser, W.W. (2002) Parasitoids induce production of the dispersal morph in the pea aphid, Acyrthosiphon pisum. Oikos 98, 323-333.

Sloggett, J.J. \& Weisser, W.W. (2004) A general mechanism for predator- and parasitoid-induced dispersal in the pea aphid, Acyrthosiphon pisum (Harris). pp. 79-85 in 6th International Symposium on Aphids, 3-7 September 2001, Rennes, France.

Sutherland, O.R.W. (1969a) The role of crowding in the production of winged forms by two strains of the pea aphid, Acyrthosiphon pisum. Journal of Insect Physiology 15, 1385-1410.

Sutherland, O.R.W. (1969b) The role of the host plant in the production of winged forms by two strains of the pea aphid Acyrthosiphon pisum. Journal of Insect Physiology 15, $2179-2201$.

Sutherland, O.R.W. \& Mittler, T.E. (1971) Influence of diet composition and crowding on wing production by the aphid Myzus persicae. Journal of Insect Physiology 17, 321-328.

Venables, W.N., Smith, D.M. \& the R Development Core Team (2002) An introduction to R. Network Theory Ltd.

Wagner, D., Tissot, M., Cuevas, W. \& Gordon, D.M. (2000) Harvester ants utilize cuticular hydrocarbons in nestmate recognition. Journal of Chemical Ecology 26, 2245-2257.

Watt, A.D. \& Dixon, A.F.G. (1981) The role of cereal growth stages and crowding in the induction of alatae in Sitobion avenae and its consequences for population growth. Ecological Entomology 6, 441-447.

Weisser, W.W., Braendle, C. \& Minoretti, N. (1999) Predatorinduced morphological shift in the pea aphid. Proceedings of the Royal Society of London Series B 266, 1175-1182.

Weisser, W.W., Minoretti, N. \& Sloggett, J.J. (2004) Wing induction by natural enemies: cues, mechanisms and adaptiver value. pp. 103-108 in 6th International Symposium on Aphids, 3-7 September 2001, Rennes, France.

(Accepted 9 November 2004)

(c) CAB International, 2005 


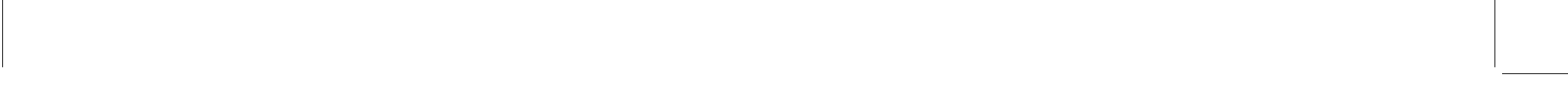

